\title{
Antifungal Activity of Extracts Biarum carduchorum (Kardeh) on Aspergillus fumigatus and Penicillium expansum in Vitro
}

\author{
Farideh Tabatabaei-Yazdi, ${ }^{1,}{ }^{*}$ Behrooz Alizadeh-Behbahani,, ${ }^{1}$ Alireza Vasiee, ${ }^{1}$ Seyed Ali Mortazavi, ${ }^{1}$ and \\ Forouzan Tabatabaei-Yazdi ${ }^{2}$ \\ ${ }^{1}$ Department of Food Science and Technology, Faculty of Agriculture, Ferdowsi University of Mashhad, Mashhad, IR Iran \\ ${ }^{2}$ Department of Animal Science, Faculty of Agriculture, Ferdowsi University of Mashhad, Mashhad, IR Iran \\ "Corresponding author: Farideh Tabatabaei-Yazdi, Department of Food Science and Technology, Faculty of Agriculture, Ferdowsi University of Mashhad, Mashhad, IR Iran. \\ E-mail: tabatabai@um.ac.ir
}

Received 2015 January 15; Accepted 2015 November 16.

\begin{abstract}
Background: Plants provide the probability of a strategy in exploration for new drugs. Infectious diseases, which account for the significant ratio of the health problems, are most frequently catered for by this system of medicine.

Objectives: The aim of this study is to investigate the antimicrobial effect of Biarum carduchorum (Kardeh) on Aspergillus fumigatus and Penicillium expansum in vitro.

Materials and Methods: In this experimental study the antibacterial activity of methanol and aqueous extracts of Biarum carduchorum against 2 laboratory strains of fungi (Aspergillus fumigatus and Penicillium expansum), were evaluated with using paper disk methods, Collins method, minimal inhibitory concentration (MIC) and minimum fungicidal concentration (MFC).

Results: The methanolic extract inhibited the growth of all tested microorganisms. The phytochemical analysis of the methanolic extract of B. carduchorum revealed the presence of phenolics (+++), alkaloids (+++), tannins (++), flavonoids (++), saponins (+), phlobatanins $(+)$, anthraquinones $(+)$, terpenes $(++)$ and cardiac glycosides $(+)$. The result showed that MIC of $B$. carduchorum leaves of the aqueous and methanolic extracts for $P$. expansum and $A$. fumigatus was 32, 8, 16, $4 \mathrm{mg} / \mathrm{mL}$ respectively. The aqueous and methanolic extracts MFC of $B$. carduchorum leaves for $P$. expansum was 64 and $32 \mathrm{mg} / \mathrm{mL}$ respectively.

Conclusions: The presence of antibacterial activity in different fractions indicates that the extract possesses different compounds, which have different activities. The result of this study suggests that the methanolic and aqueous extracts of $B$. carduchorum could be suitable for the treatment on the microorganisms associated with infections.
\end{abstract}

Keywords: Extract, Phytochemical, Antifungal, Aspergillus fumigates

\section{Background}

For a long period of time, plants have been a noteworthy source of natural products for maintaining human health, particularly in the last decade, with more severe studies for natural therapies. According to world health organization medicinal plants would be the best source to obtain a diversity of drugs [1,2].

Numerous species of the genus Biarum carduchorum have been used since ancient times in folk medicine and have been subjected to vast pharmacognostic research predesignate to identify biologically active compounds [3]. Biarum is a genus of flowering plants in the Araceae family. It is multiplex of plants that are indigenous to the southern Europe, North Africa and Middle East. The leaves of Biarum can be similar to grass or even oval. Their corms are spherical and the plants as a whole tend to be small. To flower Biarum required a dry rest period during summer. It's not fully known how they are dispersed, but one idea is that they have evolved to elude seed scattering since doing so in such inhospitable environments might not be favorable to its breeding [3].

Aspergillus fumigatus is the most repeated cause of invasive fungal infection in immunosuppressed individuals, which include patients receiving immunosuppressive therapy for autoimmune or neoplastic disease, organ transplant recipients and acquired immunodeficiency syndrome (AIDS) patients [4]. Due to the small size of conidia, many conidia deposit in alveoli where they interact with mucous and natural effectors cells. A. fumigatus primarily causes invasive infection in the lung and represents a major cause of illness and fatality in these individuals. A. fumigatus can cause chronic pulmonary infections, allergic bronchopulmonary aspergillosis, or allergic disease in immunocompetent hosts. Additionally, immunosuppressed individuals are susceptible to invasive A. fumigatus infection, which most generally manifests as invasive pulmonary aspergillosis [5].

Mycotoxicosis is the term used for poisoning linked 
with presentation to mycotoxins. These toxins can enter the blood stream and lymphatic system; they inhibit protein synthesis, damage macrophage systems, inhibit particle clearance of the lung and increase sensitivity to bacterial endotoxin [6]. The mycotoxin produced by Penicillium expansum is patulin. Patulin is a neurotoxin that can be found in apples and apple products such as juice and cider. In turn, mycotoxins have the potential for both acute and chronic health effects via ingestion, skin contact and inhalation [7].

One significant aspect of this is the emergency of resistant strains so that in spite of the efforts has been done to product broad spectrum antimicrobial substances [8]. Due to microbial resistance to common antimicrobial drugs and less toxic side effects of natural treatment such as applications of B. carduchorum extract on the face of fungal under in vitro condition looks noteworthy.

\section{Objectives}

The aim of this study was to determine antifungal effect of the aqueous and methanolic extracts of B. carduchorum (different concentrations) on A. fumigatus and P. expansum in vitro.

\section{Materials and Methods}

This experimental study was conducted at industrial microbiology laboratory, department of food science and technology, Ferdowsi university of Mashhad in 2014.

\subsection{Collection of Sample}

B. carduchorum was collected in 2014 from plants growing wild in Shir-Ali near Behbahan, Khuzestan province (south of Iran). The plant material was identified by staff at the herbarium of Ferdowsi university of Mashhad, Mashhad, Razavi Khorasan province, Iran.

\subsection{Preparation of Extract}

Percolation method was performed to prepare the extracts. Fifty grams of dried samples powder was mixed with $250 \mathrm{~mL}$ of water and methanol, and it was shaken in Erlenmeyer flask continuously for 3 minutes, then it was stored at room temperature $\left(20^{\circ} \mathrm{C}\right.$ for 48 hours). In the next step the extract was filtered through Whatman No. 1 (9 mm diameter) filter paper (Behan, Iran); the collecting supernatant was centrifuged by 2500 rpm for 15 minutes. The resulting extract (supernatant) volume has reached to the original with methanol or distilled water, and then samples were stored into the dark container at refrigerator temperature after filtering by $0.45 \mu$ Whatman filter paper [9].

\subsection{Phytochemical Screening}

The phytochemical constituents of the methanolic stem crude extracts of $B$. carduchorum were analyzed according to the methods described by Njoku and Obi [10].

\subsection{Test for Saponins}

The filtered plant extract $(0.5 \mathrm{~g})$ was mixed with few drops of $5 \% \mathrm{Na}_{2} \mathrm{CO}_{3}$ (Sodium carbonate) and few drops of Fehling's solution added and boiled. The presence of a brown precipitate indicated a positive test.

\subsection{Test for Anthraquinones}

Half a gram of the plant extract was boiled with $5 \mathrm{~mL}$ of $10 \%$ sulphuric acid $\left(\mathrm{H}_{2} \mathrm{SO}_{4}\right)$ and filtered. A pink, red or violet coloration in ammonia phase indicated a positive test [10].

\subsection{Test for Flavonoids}

Few pieces of magnesium metal strip were added to $5 \mathrm{~mL}$ of the filtrate plant extract with concentrated hydrochloric acid ( $5 \mathrm{~mL}$ ). The formation of orange, red, crimson or magenta was taken as a positive test.

\subsection{Test for Terpene}

Half a gram ( $0.5 \mathrm{~g})$ of the plant extract was dissolved in $3 \mathrm{~mL}$ of chloroform and filtered. Ten drops of acetic anhydride were added to the filtrate with 2 drops of concentrated sulphuric acid $\left(\mathrm{H}_{2} \mathrm{SO}_{4}\right)$; pink colour at the interphase was taken as the positive test.

\subsection{Test for Phenolics}

To $2 \mathrm{~mL}$ of the ethanolic extract, $1 \mathrm{~mL}$ of ferric chloride solution was added. Blue or green colour indicated a positive test.

\subsection{Test for Phlobatanins}

This was carried out by boiling $0.5 \mathrm{~mL}$ of the extract mixture with $5 \mathrm{~mL}$ of water and $1 \% \mathrm{HCl}$ in a test tube for 2 minutes. The colour change was regarded as positive for phlobatanins [10].

\subsection{Test for Alkaloids}

The plant extract $(0.5 \mathrm{~g})$ was added with a few drops of picric acid reagent. A white or yellow precipitate indicated a positive test.

\subsection{Test for Tannins}

Half a gram ( $0.5 \mathrm{~g})$ of the filtered plant extract was put in a test tube and $9 \mathrm{~mL}$ of distilled water added. Decolouration observed upon addition of bromine water indicated positive test for tannin [10]. 


\subsection{Test Organisms}

Two bacteria were tested in this study include; $A$. fumigatus ATTC 1022 and $P$. expansum ATTC 24692 were obtained from the microbiology department of Mashhad University of Medical Sciences, Mashhad.

\subsection{Inoculum Preparation}

Molds A. fumigatus and P. expansum were maintained on Sabouraud dextrose agar (Merck, Germany). Sterile distilled water containing $0.05 \%$ Tween 80 was added to the surface growth and spores and hyphae were scraped off with a sterile wire loop. Sterile tubes containing Ringer solution and its turbidity, was measured by spectrophotometer at $530 \mathrm{~nm}$ wavelength. It was diluted by Ringer solution until the solution turbidity equalizes with 0.5 McFarland standard solutions. Suspension should have contains $1.5 \times$ $10^{8} \mathrm{CFU} / \mathrm{mL}[11,12]$.

\subsection{Antimicrobial Activity}

The agar disc diffusion method was employed to determine antifungal activity in this study. For agar disc diffusion method, the disc ( $7 \mathrm{~mm}$ ) on Sabouraud Dextrose Agar (SDA) (Merck) plates were saturated with $100 \mu \mathrm{L}$ of the test compound allowed to dry and was introduced on the upper layer of the seeded agar plate. The plates were incubated 72 hours at $27^{\circ} \mathrm{C}$. Microbial growth was determined by measuring the diameter of inhibition zone. For each fungi strain, controls were maintained where pure solvents were used instead of the extract. The result was obtained by measuring the zone diameter. The experiment was done three times and the mean values are presented [13].

\subsection{Determination of MIC}

MIC determination was performed using the serial dilutions method. At first, suspension of A. fumigatus and $P$. expansum according to standard 0.5 McFarland was provided in Sabouraud Dextrose Broth (SDB) (Merck, Germany). One millimeter of SDB was added to them. In the next step $1 \mathrm{~mL}$ of extract was added to tubes according to serial dilutions procedure. Also $20 \mu \mathrm{L}$ of $A$. fumigatus and $P$. expansum suspension was added to tubes. Then they were incubated in $35^{\circ} \mathrm{C}$ for 24 hours. Tube number 10 was considered as the control tube $(1 \mathrm{~mL} \mathrm{SDB}+20 \mu \mathrm{L}$ A. fumigatus and $P$. expansum suspension). After incubation, with observation of turbidity or lack of turbidity in tubes, level of MIC was determined [14].

\subsection{Determination of $M F C$}

MFC was determined according to agar dilution method [15] with slight modifications. The SDB without incorporation of dried herbal extract and $1 \mathrm{~mL}$ of adjusted spore suspension served as positive control and SDB alone served as negative control. The tubes which showed no visible growth after 3 days incubation were subculture on extract free SDA plates and incubated at $27^{\circ} \mathrm{C}$ for 7 days. The MFC was regarded as the lowest concentration of the extract that prevented the growth of any molds colony on the solid medium.

\subsection{Statistical Analysis}

In order to analyze the information, descriptive and inferential methods were utilized. The test results were processed by using one-way variance analysis (ANOVA). All of our analysis was done by SPSS-18. $\mathrm{P}<0.05$ were considered statistically significant.

\section{Results}

The results of antifungal effects $B$. carduchorum methanolic extract in screening antimicrobial activity method were showed that B. carduchorum extracted in screening antimicrobial activity method in $2000 \mu \mathrm{g} / \mathrm{mL}$, inhibit A. fumigatus and P. expansum growth (Figure 1 and Table 1). The results of antifungal activity of $B$. carduchorum aqueous extract in screening antimicrobial activity method were showed that the $B$. carduchorum extract in $2000 \mu \mathrm{g} / \mathrm{mL}$, had no significant antifungal effect on $A$. fumigatus and $P$. expansum and it is not able to prevent the growth of fungi on culture (Figure 1 and Table 1 ).

Table 1. Antimicrobial Effects of Ethanolic and Aqueous Biarum carduchorum Extracts Concentrations on Aspergillus fumigatus and Penicillium expansum

\begin{tabular}{lcc}
\hline Extract & Microorganism & Result \\
\hline Aqueous & Aspergillus fumigatus ATTC 1022 & $\mathrm{R}$ \\
Aqueous & Penicillium expansum ATTC 24692 & $\mathrm{R}$ \\
\hline Methanolic & Aspergillus fumigatus ATTC 1022 & $\mathrm{S}$ \\
Methanolic & Penicillium expansum ATTC 24692 & $\mathrm{S}$ \\
\hline
\end{tabular}

The results of determination dry weight of extracts were shown in (Figure 2). The result shows that dry weight of ethanolic extract of $B$. carduchorum $13 \%$ and for aqueous was $9 \%$. The results indicate that methanolic extract of $B$. carduchorum $4 \%$ mostly aqueous extract.

The result shows that the $B$. carduchorum methanolic extracts had inhibition effect on both A. fumigatus and $P$. expansum (in 15, 30, 45 and $60 \mathrm{mg} / \mathrm{mL}$ ) but the B. carduchorum aqueous extract had inhibition effect on A. fumigatus 
Figure 1. Antifungal Activity of Methanolic and Aqueous Biarum Carduchorum Extract on Aspergillus fumigatus and/or Penicillium expansum (Screening for Antimicrobial Activity)
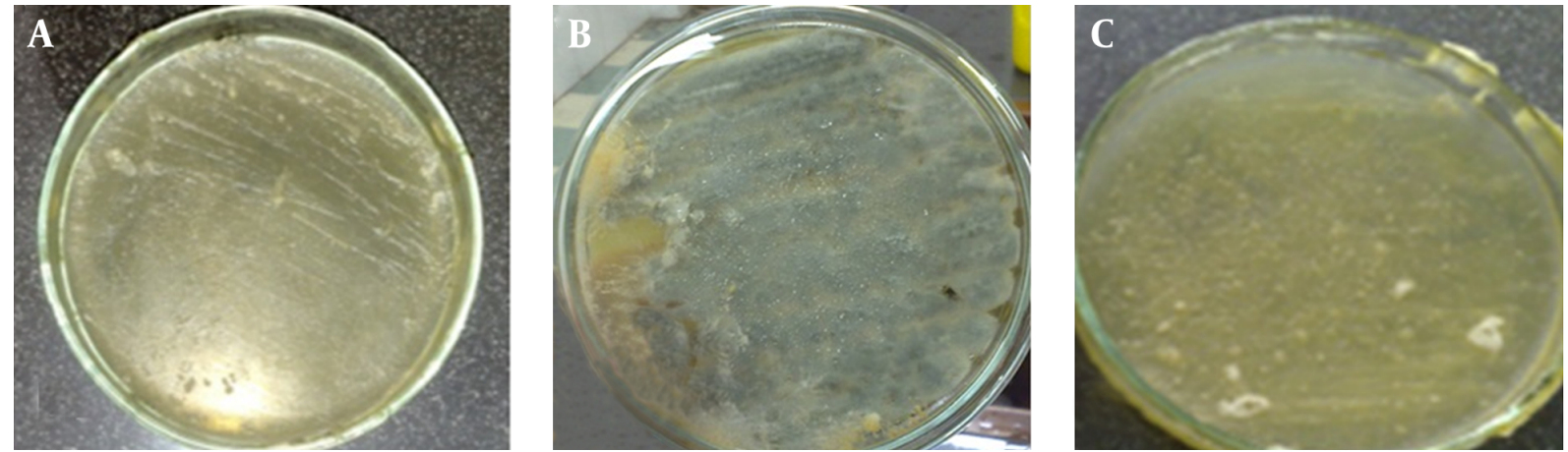

A, Antimicrobial effects of $2000 \_g / m L$ Biarum carduchorum extract concentrations (methanolic); B, Control Penicillium expansum; C, Antimicrobial effects of 2000_g/mL Biarum carduchorum extract concentrations (methanolic)

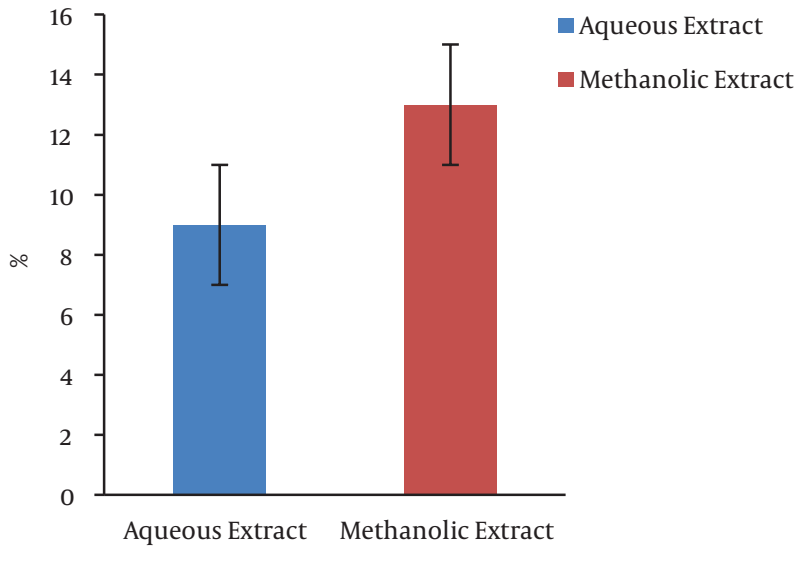

Figure 2. Dry Weight of Extracts

(in 15, 30, 45 and $60 \mathrm{mg} / \mathrm{mL}$ ) and P. expansum (in 30, 45 and $60 \mathrm{mg} / \mathrm{mL}$ ) (Table 2 and Figure 3 ).

The result shows that MIC of B. carduchorum methanolic extract for A. fumigatus and P. expansum was 4 and 16 $\mathrm{mg} / \mathrm{mL}$ respectively. The results show that MIC of aqueous extract of B. carduchorum for A. fumigatus was $8 \mathrm{mg} / \mathrm{mL}$, and for $P$. expansum was $32 \mathrm{mg} / \mathrm{mL}$ (Table 3 ).

The result shows that MFC of aqueous extract of B. carduchorum for A. fumigatus was $32 \mathrm{mg} / \mathrm{mL}$, and for P. expansum was $64 \mathrm{mg} / \mathrm{mL}$. The results show that MFC of methanolic extract of B. carduchorum for A. fumigatus was $16 \mathrm{mg} / \mathrm{mL}$, and for $P$. expansum was $32 \mathrm{mg} / \mathrm{mL}$ (Table 4 ).

The results shows that phytochemical analysis of the extract of B. carduchorum revealed the presence of phenolics (+++), alkaloids (+++), tannins (++), flavonoids (++), saponins $(+)$, phlobatanins $(+)$, anthraquinones $(+)$, ter-

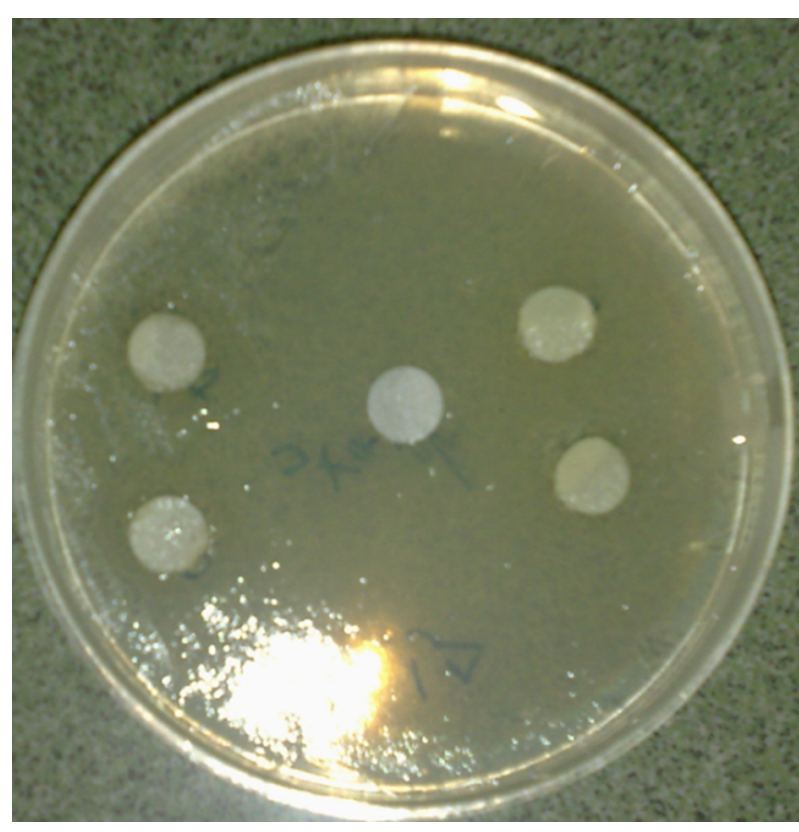

Figure 3. Average Diameter (mm) of Microbial Free Zone Area of Biarum carduchorum Extract on Penicillium expansum

penes $(++)$ and cardiac glycosides $(+)$ (Table 5).

\section{Discussion}

The results of antifungal activity of B. carduchorum aqueous extract in screening antimicrobial activity method were showed that the $B$. carduchorum extract in $2000 \mu \mathrm{g} / \mathrm{mL}$, had no significant antifungal effect on $A$. fumigatus and $P$. expansum and it is not able to prevent 
Table 2. Average Diameter (mm) of Microbial Free Zone Area of Methanolic and Aqueous Biarum carduchorum Extracts on Aspergillus fumigatus and Penicillium expansum (Disk Agar Diffusion Method), $(\mathrm{n}=3)^{\mathrm{a}}$

\begin{tabular}{|c|c|c|c|c|}
\hline \multirow[t]{2}{*}{ Fungi Species } & \multicolumn{4}{|c|}{ Concentration, $\mathbf{m g} / \mathbf{m L}$} \\
\hline & 15 & 30 & 45 & 60 \\
\hline Methanolic Aspergillus fumigatus & $9.6 \pm 0.28$ & $11.5 \pm 0.50$ & $13.6 \pm 0.58$ & $16.2 \pm 0.58$ \\
\hline Methanolic Penicillium expansum & $8.4 \pm 0.29$ & $11 \pm 0.58$ & $13 \pm 0.28$ & $15 \pm 0.54$ \\
\hline Aqueous Aspergillus fumigatus & $8.1 \pm 0.28$ & $10.3 \pm 0.50$ & $11.6 \pm 0.54$ & $13.8 \pm 0.54$ \\
\hline
\end{tabular}

Table 3. Minimum Inhibitory Concentration (MIC) of Methanolic and Aqueous Biarum carduchorum Extract on Aspergillus fumigatus and Penicillium expansum

\begin{tabular}{|c|c|c|c|c|c|c|c|c|c|c|}
\hline \multirow[t]{2}{*}{ Fungi Species } & \multicolumn{9}{|c|}{ Concentration, mg/mL } & \multirow[b]{2}{*}{ Negative } \\
\hline & 2 & 4 & 8 & 16 & 32 & 64 & 128 & 256 & Positive & \\
\hline Methanolic Aspergillus fumigatus & + & . & - & - & - & - & - & - & + & - \\
\hline Methanolic Penicillium expansum & + & + & + & - & - & . & - & - & + & . \\
\hline Aqueous Aspergillus fumigatus & + & + & - & . & - & . & - & . & + & - \\
\hline Aqueous Penicillium expansum & + & + & + & + & . & . & . & . & + & . \\
\hline
\end{tabular}

Table 4. Minimum Fungicidal Concentration (MFC) of Methanolic and Aqueous Biarum carduchorum extract on Aspergillus fumigatus and Penicillium expansum, $(\mathrm{n}=3)^{\mathrm{a}}$

\begin{tabular}{|c|c|c|c|c|c|c|c|c|c|c|}
\hline \multirow[t]{2}{*}{ Fungi Species } & \multicolumn{9}{|c|}{ Concentration, $\mathrm{mg} / \mathrm{mL}$} & \multirow[b]{2}{*}{ Negative } \\
\hline & 2 & 4 & 8 & 16 & 32 & 64 & 128 & 256 & Positive & \\
\hline Methanolic Aspergillus fumigatus & + & + & + & - & $\cdot$ & $\cdot$ & $\cdot$ & - & + & - \\
\hline Methanolic Penicillium expansum & + & + & + & + & - & - & - & - & + & - \\
\hline Aqueous Aspergillus fumigatus & + & + & + & + & . & . & . & . & + & . \\
\hline Aqueous Penicillium expansum & + & + & + & + & + & . & . & . & + & . \\
\hline
\end{tabular}

$\mathrm{a}_{+: \text {Grow, }: \text { Not grow. }}$

Table 5. Phytochemical Constituents of the Extracts of Biarum carduchorum

\begin{tabular}{lc}
\hline Plant Constituents & Occurrences \\
\hline Phenolics & +++ \\
Alkaloids & +++ \\
\hline Tannins & ++ \\
\hline Flavonoids & ++ \\
\hline Saponins & + \\
\hline Phlobatanins & + \\
\hline Anthraquinones & ++ \\
\hline Terpenes & + \\
\hline Cardiac glycosides & + \\
\hline Glycosides & + \\
\hline
\end{tabular}

${ }^{a}+$ : Present in small concentrations, ++: present in moderately high concentrations, +++: present in very high concentrations. the growth of fungi on culture. The methanolic extract inhibited the growth of all tested microorganisms. The phytochemical analysis of the methanolic extract of $B$. carduchorum revealed the presence of phenolics $(+++)$, alkaloids (+++), tannins (++), flavonoids (++), saponins $(+)$, phlobatanins $(+)$, anthraquinones $(+)$, terpenes $(++)$ and cardiac glycosides $(+)$. The result showed that MIC of B. carduchorum leaves of the aqueous and methanolic extracts for $P$. expansum and A. fumigatus was $32,8,16,4 \mathrm{mg} / \mathrm{mL}$ respectively. The aqueous and methanolic extracts MFC of B. carduchorum leaves for P. expansum was 64 and $32 \mathrm{mg} / \mathrm{mL}$ respectively. It is important to study scientifically plants that have been used in traditional medicines to determine potential sources of novel antimicrobial compounds [16].

Plants are rich in a wide variety of phytochemicals like tannins, terpenoids, alkaloids, flavonoids, antimicrobial peptides, etc., that have been found to have antimicrobial activities. The effectiveness of the active compounds present in plant extracts cause the production of growth inhibition zones that appear as clear areas surrounding 
the disk. Antifungal activity may be due to active components which are present in plant extracts. However, some plant extracts were unable to exhibit antimicrobial activity against tested fungi strains. These fungi strains may have some kind of resistance mechanisms e.g. enzymatic inactivation, target sites modification and decrease intracellular drug accumulation. The use of plant extracts in the treatment of diseases caused by various bacteria, viruses and fungi were reported. Antifungal properties of plant extracts are widely recognized [17].

In this study, by increasing the amount of ethanolic extract on disc diffusion method, inhibition zone around the disc was increased. The result shows that the $B$. carduchorum methanolic extracts had inhibition effect on both A. fumigatus and P. expansum (in 15, 30, 45 and $60 \mathrm{mg} / \mathrm{mL}$ ) but the $B$. carduchorum aqueous extract had inhibition effect on A. fumigatus (in 15, 30, 45 and $60 \mathrm{mg} / \mathrm{mL}$ ) and $P$. expansum (in 30, 45 and $60 \mathrm{mg} / \mathrm{mL}$ ). The traditional healers use primarily water as the solvent but we found in this study the plant extracts by methanol provided more consistent antimicrobial activity compared to those extracted by water. This might have resulted from the lack of solubility of the active constituents in aqueous solutions while methanol extract showed some degree of antibacterial activity. Further trials using solvents of various polarities will explore the effects of solvent composition on extract efficacy. Alizadeh-Behbahani and Tabatabaei-Yazdi reported that most of the antimicrobial active compounds were soluble in polar solvent such as ethanol instead of water [13].

Alizadeh-Behbahani et al. antifungal effects of extract were determined by screening antimicrobial activity and disk agar diffusion test in 20, 40, 60 and $80 \mathrm{mg} / \mathrm{mL}$ concentration of the extract against $P$. digitatum and $A$. citri. The results indicate that extracts of A. marina mostly had been effective on $P$. digitatum and have the least impact on A. citri. The results show that the ethanol extract of mangrove leaf with antimicrobial activity method in $2 \mathrm{mg} / \mathrm{mL}$, were inhibited the growth of $P$. digitatum and A. citri. In disk agar diffusion test the mentioned extract were shown inhibition effect on pathogen molds growth [18]. Concern regarding the development of resistance to any of the few antifungal drugs available has developed. Although we are able to define certain mechanisms of drug resistance, continued efforts for a deeper understanding of the cellular and molecular mechanisms as well as the clinical components of antifungal resistance will be important [19]. The result shows that MIC of B. carduchorum leaves methanolic extract for A. fumigatus and P. expansum was 4 and 16 $\mathrm{mg} / \mathrm{mL}$ respectively. The results show that MIC of aqueous extract of B. carduchorum for A. fumigatus was $8 \mathrm{mg} / \mathrm{mL}$, and for P. expansum was $32 \mathrm{mg} / \mathrm{mL}$. The results of this study showed that the $B$. carduchorum extract show antifungal properties justify their traditional use as medicinal plants. In conclusion, $B$. carduchorum plants may have potential medicinal importance, and could suggest that $B$. carduchorum extract in vitro, have considerable antimicrobial ability over the studied strains. The presence of antibacterial activity in different fractions indicates that the extract possesses different compounds, which have different activities. The result of this study suggests that the methanolic and aqueous extracts of $B$. carduchorum could be suitable for the treatment on the microorganisms associated with infections. Tabatabaei-Yazdi et al. investigated the antimicrobial effect of ethanol and aqueous extracts of Dorema aucheri (Bilhar) against some pathogenic bacterial strains including 4 Gram positive (Staphylococcus aureus, Streptococcus pyogenes, Bacillus cereus and Bacillus subtilis) and 3 gram negative bacteria (Escherichia coli, Pseudomonas aeruginosa and Proteus vulgaris [2].

The results showed that MIC of the extracts ranged between $2 \mathrm{mg} / \mathrm{mL}$ and $64 \mathrm{mg} / \mathrm{mL}$ while the minimum lethal concentration (MLC) ranged between $4 \mathrm{mg} / \mathrm{mL}$ and 256 $\mathrm{mg} / \mathrm{mL}$. Among of tested strains, $P$. aeruginosa has maximum MIC and MBC. Thirty and $40 \mathrm{mg} / \mathrm{mL}$ concentrations of redcurrant have significant antimicrobial effect on bacteria.

The present study demonstrated that the ethanol leaf extract of B. carduchorum hold an excellent potential as an antibacterial agent. Further in vivo studies are necessary. More importantly there is need for detailed scientific study of traditional medical practices to ensure that valuable therapeutic knowledge of some plants is preserved and also to provide scientific evidence for their efficacies.

\section{Acknowledgments}

The authors wish to express their profound gratitude and thank sincerely to research's deputy of Ferdowsi University of Mashhad for providing the cost of this project with code $2 / 32215$.

\section{Footnotes}

Authors' Contribution: Farideh Tabatabaei-Yazdi, Behrooz Alizadeh-Behbahani, Alireza Vasiee, Mortazavi Seyed Ali Mortazavi, Forouzan Tabatabaei-Yazdi for conceptualisation of study and experimental design; standardization and optimisation of all protocols; Data acquisition, analysis and interpretation; Manuscript write and final approval of the submitted version.

Conflict of Interest: The authors declare no conflict of interest. 
Funding/Support: Funding for this work was provided by the research council of the Ferdowsi university of Mashhad.

\section{References}

1. Khan MA, Ashfaq MK, Zuberi HS, Mahmood MS, Gilani AH. The in vivo antifungal activity of the aqueous extract from Nigella sativa seeds. Phytother Res. 2003;17(2):183-6. doi: 10.1002/ptr.1146. [PubMed: 12601685].

2. Tabatabaei-Yazdi F, Alizadeh-Behbahani B, Vasiee A, Mortazavi SA, abatabaei Yazdi F. An investigation on the effect of alcoholic and aqueous extracts of Dorema aucheri (Bilhar) on some pathogenic bacteria in vitro. J Paramed Sci. 2015;6(1):58-64.

3. Boyce PC.A Taxonomic Revision of Biarum.Curtis's Botanical Magazine. 2008;25(1):2-17. doi: 10.1111/j.1467-8748.2007.00607.x.

4. Nierman WC, Pain A, Anderson MJ, Wortman JR, Kim HS, Arroyo J, et al. Genomic sequence of the pathogenic and allergenic filamentous fungus Aspergillus fumigatus. Nature. 2005;438(7071):1151-6. doi: 10.1038/nature04332. [PubMed:16372009].

5. Latge JP. The pathobiology of Aspergillus fumigatus. Trends in Microbiology. 2001;9(8):382-9. doi: 10.1016/s0966-842x(01)02104-7.

6. Raju MV, Devegowda G. Influence of esterified-glucomannan on performance and organ morphology, serum biochemistry and haematology in broilers exposed to individual and combined mycotoxicosis (aflatoxin, ochratoxin and T-2 toxin). Br Poult Sci. 2000;41(5):640-50 doi: 10.1080/713654986. [PubMed: 11201446].

7. Andersen B, Smedsgaard J, Frisvad JC. Penicillium expansum: consistent production of patulin, chaetoglobosins, and other secondary metabolites in culture and their natural occurrence in fruit products. JAgric Food Chem. 2004;52(8):2421-8. doi:10.1021/jf035406k. [PubMed: 15080656].

8. Goossens $\mathrm{H}$, Ferech $\mathrm{M}$, Vander Stichele R, Elseviers $\mathrm{M}$, Esac Project Group . Outpatient antibiotic use in Europe and association with resistance: a cross-national database study. Lancet.
2005;365(9459):579-87. doi:10.1016/S0140-6736(05)17907-0. [PubMed: 15708101].

9. Hsieh P, Mau J, Huang S. Antimicrobial effect of various combinations of plant extracts. Food Microbiology. 2001;18(1):35-43. doi: 10.1006/fmic.2000.0376.

10. Njoku VO, Obi C. Phytochemical constituents of some selected medicinal plants. Afri J Pure Appl Chem. 2009;3(11):228-33.

11. Saetae D, Suntornsuk W. Antifungal activities of ethanolic extract from Jatropha curcas seed cake.J Microbiol Biotechnol. 2010;20(2):31924. [PubMed: 20208435].

12. Collins C, Grange J, Lyne P. Collins and Lyne's microbiological methods. 8 ed. USA: CRC Press; 2004.

13. Alizadeh-Behbahani B, Tabatabaei-Yazdi F. In vitro study of antibacterial activity of Mangle negro extracts against selected pathogens from enterobacteriaceae and bacillaceae familia. Zahedan J Res Med Sci.

14. Alizadeh Behbahani B, Tabatabaei Yazdi F, Noorbakhsh H, Riazi F, Jajarmi A, Tabatabaei Yazdi F. Study of the Antibacterial Activity of Methanolic and Aqueous Extracts of Myrtus communis on Pathogenic Strains Causing Infection. Zahedan J Res Med Sci. 2016;In Press(InPress) doi: 10.17795/zjrms-5989.

15. Tabatabaei-Yazdi F, Alizadeh-Behbahani B, Zanganeh H. The comparison of antibacterial activity of Mespilus germanica extracts with a variety of common therapeutic antibiotics In Vitro. Zahedan J Res Med Sci. .

16. Dzoyem JP, Tangmouo JG, Lontsi D, Etoa FX, Lohoue PJ. In vitro antifungal activity of extract and plumbagin from the stem bark of Diospyros crassiflora Hiern (Ebenaceae). Phytother Res. 2007;21(7):671-4. doi: 10.1002/ptr.2140. [PubMed:17444575].

17. Stevens DA, Holmberg K. Resistance to antifungal drugs: current status and clinical implications. Curr Opin Anti Infect Invest Drugs. 1999:306-17.

18. Alizadeh Behbahani B, Tabatabaei Yazdi F, Shahidi F, Riazi F. Antifungal Effect of the Aqueous and Ethanolic Avicennia marina Extracts on Alternaria citri and Penicillium digitatum. Zahedan J Res Med Sci. 2016;In Press(InPress) doi: 10.17795/zjrms-5992.

19. Rani S, Murty S. Antifungal potential of flower head extract of Spilanthes acmella Linn. Afr J Biomed Res. 2006;9(1):67-9. 